\title{
Abnormal Heart Rate Characteristics Preceding Neonatal Sepsis and Sepsis-Like Illness
}

\author{
M. PAMELA GRIFFIN, T. MICHAEL O'SHEA, ERIC A. BISSONETTE, \\ FRANK E. HARRELL, JR., DOUGLAS E. LAKE, AND J. RANDALL MOORMAN

\begin{abstract}
Departments of Pediatrics [M.P.G.], Health Evaluation Sciences [E.A.B., F.E.H.], Internal Medicine [D.E.L., J.R.M.], and Molecular Physiology and Biological Physics [J.R.M.], and the Cardiovascular Research Center, University of Virginia Health System, Charlottesville, Virginia 22908, U.S.A.; and

Department of Pediatrics [T.M.O.], Wake Forest School of Medicine,

Winston-Salem, North Carolina 27157, U.S.A.
\end{abstract}

\begin{abstract}
ABSTR
Late-onset neonatal sepsis is a significant cause of morbidity
and mortality, and early detection could prove beneficial. Previ-
ously, we found that abnormal heart rate characteristics (HRC) of
reduced variability and transient decelerations occurred early in
the course of neonatal sepsis and sepsis-like illness in infants in
a single neonatal intensive care unit (NICU). We hypothesized
that this finding can be generalized to other NICUs. We prospec-
tively collected clinical data and continuously measured RR
intervals in all infants in two NICUs who stayed for $>7$ d. We
defined episodes of sepsis and sepsis-like illness as acute clinical
deteriorations that prompted physicians to obtain blood cultures
and start antibiotics. A predictive statistical model yielding an
HRC index was developed on a derivation cohort of 316 neo-
nates in the University of Virginia NICU and then applied to the
validation cohort of 317 neonates in the Wake Forest University
NICU. In the derivation cohort, there were 155 episodes of sepsis
and sepsis-like illness in 101 infants, and in the validation cohort,
there were 118 episodes in 93 infants. In the validation cohort,
the HRC index 1 ) showed highly significant association with
\end{abstract}
impending sepsis and sepsis-like illness (receiver operator characteristic area $0.75, p<0.001$ ) and 2) added significantly to the demographic information of birth weight, gestational age, and days of postnatal age in predicting sepsis and sepsis-like illness $(p<0.001)$. Continuous HRC monitoring is a generally valid and potentially useful noninvasive tool in the early diagnosis of neonatal sepsis and sepsis-like illness. (Pediatr Res 53: 920-926, 2003)
BW, birth weight
Abbreviations
GA, gestational age
HRC, heart rate characteristics
HRV, heart rate variability
NICU, neonatal intensive care unit
ROC, receiver operating characteristic
SampEn, sample entropy
UVA, University of Virginia
WFU, Wake Forest University

Late-onset nosocomial neonatal sepsis occurs after 3 days of life and is a major health problem in high-risk newborn infants

Received July 4, 2002; accepted October 15, 2002.

Correspondence: M. Pamela Griffin, M.D., Department of Pediatrics, Box 800386, University of Virginia, Charlottesville, VA 22908, U.S.A.; e-mail: mpg7u@virginia.edu American Heart Association, Mid-Atlantic Consortium; Children's Medical Center Research Fund, University of Virginia; W.S. Paley Foundation; Virginia's Center for Innovative Technology; Medical Decision Networks, Charlottesville, VA; NIGMS-64640. Presented in part at the Pediatric Academic Societies' 2001 Annual Meeting.

Potential for conflict of interest: Medical Decision Networks of Charlottesville, VA, has a license to market technology related to HRC monitoring of newborn infants and supplied partial funding for this study. (Other sponsors are NIGMS 64640, the American Heart Association Mid-Atlantic Research Consortium, the Children's Medical Center of the University of Virginia, and Virginia's Center for Innovative Technology.) As of this printing, none of the authors has received consultants' fees or own equity in Medical Decision Networks or related companies. M.P.G. and J.R.M.. however, have been offered an equity share of a new company, Medical Predictive Science Corporation, that will own the HRC technology license.

DOI: 10.1203/01.PDR.0000064904.05313.D2 in the neonatal intensive care unit (NICU). It occurs in $25 \%$ of very low birth weight $(<1500 \mathrm{~g})$ infants and leads to a more than doubling of mortality and a 50\% increase in hospital stay (1). Early detection is very difficult using clinical signs and currently available laboratory tests $(2,3)$. Clinicians have found neither to be reliable $(4,5)$, and the diagnostic challenge is manifest in the Centers for Disease Control and Prevention's definition that allows diagnosis of neonatal "clinical sepsis" with either a negative blood culture or no blood culture at all (6). These high-stakes uncertainties lead to two shortcomings of the current practice. First, infants with sepsis are often detected only when seriously ill, increasing morbidity and mortality and lowering the chance for prompt, complete recovery with antibiotic therapy. Second, physicians have a very low threshold for suspecting sepsis, and as many as 10-20 infants are treated unnecessarily for every infant who truly is septic (3-5). The National Institute of Child Health and Human 
Development Neonatal Research Network concluded that successful surveillance strategies that lead to an earlier diagnosis of sepsis are urgently needed for very low birth weight infants to decrease mortality and morbidity (1).

We have found that abnormal heart rate characteristics (HRC) of reduced variability and decelerations were present in some infants for $12-24 \mathrm{~h}$ before the clinical diagnosis of sepsis (7). Interestingly, we found no difference between infants with positive ("sepsis") and negative ("sepsis-like illness") blood cultures. We interpreted the findings to be the result of a systemic inflammatory response syndrome $(8,9)$. Specifically, we hypothesized that cytokines released in response to infectious or noninfectious agents led to altered intracellular signal transduction processes. In our view, HRC analysis allows detection of these effects in real time because sinus node cells are affected like any others. Another nonexclusive point of view is that sepsis influences multiple points in the feedback control loop for heart rate variability (HRV) and that the observed changes are due to altered regulation of the heart beat by the central and autonomic nervous systems.

The goals of this study were to derive and to validate multivariable statistical models based on HRC to predict impending sepsis and sepsis-like illness. Previously, we used the measures of moments and percentiles of RR intervals, the times between heartbeats, to quantify the abnormality. Since our previous report, we have developed and evaluated two new measures of neonatal HRC. Sample entropy (SampEn) describes regularity and also detects time series with spikes (here, the heart rate decelerations) $(10,11)$. Sample asymmetry reports on asymmetry of distributions of RR intervals (Kovatchev B, Farhy L, Lake DE, Griffin MP, Moorman JR, Sample asymmetry analysis of neonatal heart rate characteristics prior to sepsis and sepsis-like illness. Pediatr Res, submitted) and is similar mathematically to the low blood glucose index developed by Kovatchev et al. (13). Here we describe the derivation and validation of regression models to predict neonatal sepsis and sepsis-like illness. Models were derived using data collected at the University of Virginia (UVA) and were then validated with data from Wake Forest University (WFU).

\section{METHODS}

Patients. We prospectively and continuously collected data on RR intervals for all infants admitted to the NICUs at UVA and WFU between September 1999 and March 2001. Both NICUs are tertiary care facilities with approximately 500 admissions per year. The UVA NICU admits both inborn infants and those transported from referral hospitals, whereas with rare exceptions, all infants admitted to the WFU NICU are born at other hospitals. The Institutional Review Boards at both institutions approved this study. Because this was a noninterventional, observational study, written informed consent was not required.

Definition of Outcome of Interest. We defined episodes of sepsis and sepsis-like illness as acute clinical deteriorations that prompted physicians to obtain blood cultures and start antibiotics. For this study that focused on late-onset sepsis, we analyzed data obtained after $7 \mathrm{~d}$ of age.

Data Acquisition. We prospectively collected clinical and $\mathrm{HRC}$ data for the entire hospital course of each infant. Clinical data included birth weight (BW), gestational age (GA), and the times and results of blood cultures obtained for the clinical diagnosis of sepsis and sepsis-like illness. HRC data were collected on microcomputers as previously described (7) and included SD, SampEn $(10,11)$, and sample asymmetry analysis of consecutive 4096-beat segments. Sample asymmetry, like skewness, measures the asymmetry of the histogram of RR intervals. It is conceptually similar to the low blood glucose index, a predictor of the risk for severe hypoglycemia in patients with type 1 diabetes (13). It yields two measures that can be directly interpreted as representing the frequency and extent of heart rate accelerations and decelerations, respectively (Kovatchev B, Farhy L, Lake DE, Griffin MP, Moorman JR, Sample asymmetry analysis of neonatal heart rate characteristics prior to sepsis and sepsis-like illness. Pediatr Res, submitted).

Data Analysis. HRC data were grouped into 6-h epochs beginning each midnight. Every $6 \mathrm{~h}$, HRC measures for the previous $12 \mathrm{~h}$ were summarized using median or 10th percentile values. The 6-h epoch containing the clinical event was not analyzed. The 14-d epoch after the event was also excluded to allow recovery of the infant and to ensure independence of any subsequent events. For the purpose of logistic regression, the HRC results for the four 6-h epochs leading up the event epoch were classified as outcome $=1$, and all others were classified as outcome $=0$.

Statistical Analysis. The mathematical and statistical analysis was carried using S-Plus (Insightful), Matlab (MathWorks), and our own programs in $\mathrm{C}++$. The statistical significance of differences in demographic features and HRC at the two sites (Table 1) was tested using the Wilcoxon rank-sum

Table 1. Demographic characteristics and HRC of the study population

\begin{tabular}{lclc}
\hline & $\begin{array}{c}\text { UVA } \\
(n=316 \text { infants })\end{array}$ & $\begin{array}{c}\text { WFU } \\
(n=317 \text { infants })\end{array}$ & $p$ \\
\hline All infants in the study & & & \\
BW (g) & $1746(1102,2852)$ & $1790(870,2751)$ & NS* \\
$<1500 \mathrm{~g}$ & $139(44 \%)$ & $131(41 \%)$ & $\mathrm{NS}^{*}$ \\
GA (wk) & $32(28,37)$ & $33(27,37)$ & $\mathrm{NS}$ \\
Mortality, overall & $19(6 \%)$ & $32(10 \%)$ & $\mathrm{NS}$ \\
Mortality, no event & $12(6 \%)$ & $13(6 \%)$ & $\mathrm{NS}$ \\
SD (ms) & 7.6 & 6.5 & $<0.001$ \\
Sample asymmetry & 0.97 & 0.99 & $\mathrm{NS}$ \\
SampEn & 0.73 & 0.53 & $<0.0001$ \\
HRC index & 3.5 & 6.8 & $<0.0001$ \\
Infants with events & $n=101(32 \%)$ & $n=93(29 \%)$ & \\
Events & 155 & 118 & $\mathrm{NS}$ \\
BW & $1010(742,1424)$ & $803(650,1488)$ & $\mathrm{NS}$ \\
$<1500 \mathrm{~g}$ & $77(76 \%)$ & $70(75 \%)$ & $\mathrm{NS}$ \\
GA & $27(25,31)$ & $26(24,30)$ & $\mathrm{NS}$ \\
$(+)$ blood cultures & $32(21 \%)$ & $27(23 \%)$ & $\mathrm{NS}$ \\
$(-)$ blood cultures & $123(79 \%)$ & $91(77 \%)$ & $\mathrm{NS}$ \\
Mortality & $7(7 \%)$ & $19(20 \%)$ & 0.01 \\
\hline
\end{tabular}

Data are presented as median (25th, 75 th percentile).

$*$ Cutoff $p \leq 0.05$. 
test.

We developed predictive statistical models based on logistic regression on a derivation cohort in the UVA NICU and then tested the models on a validation cohort at WFU. We refer to models that use nonchanging or slowly changing variables (i.e. the demographic features BW, GA, and days of postnatal age) as static and models that use the more quickly changing HRC measures as dynamic.

Repeated measures logistic regression models $(14,15)$ were built using incidence of a blood culture as a response with days of postnatal age (age), GA, BW, and repeated HRC measurements as independent predictors. Nonlinear terms for age were included using a cubic spline function with six knots tested for sufficiency using the Akaike Information Criterion. The covariance matrix of the model coefficients was corrected for correlated responses from the same infant using the Huber-White method (16). Models were summarized and compared using Wald statistics and area under the receiver operating characteristic (ROC) curve. We obtained final models using the Akaike Information Criterion and penalized maximum likelihood techniques to shrink the regression coefficients to the level of complexity supported by the data (15).

We define the HRC index to be the output of a regression model that used only HRC to predict sepsis and sepsis-like illness. It is a unitless number between 0 and 1 that is proportional to the risk of an acute clinical deterioration in the next $24 \mathrm{~h}$. We quantified the incremental gain in using the HRC index over clinical parameters alone in a test set of data using the techniques outlined above where the log-odds of developing sepsis over the next $24 \mathrm{~h}$ was modeled as the logit, or linear combination of terms:

$\beta_{0}+\beta_{1}$ HRC index $+\beta_{2} \mathrm{GA}+\beta_{3} \mathrm{BW}+\beta_{4}$ Age + nonlinear terms for age

The null hypothesis that the HRC index provided no incremental diagnostic information over clinical parameters is $\mathrm{H}_{0}$ : $\beta_{1}=0$. The $p$ value of this hypothesis was calculated using a Wald test. The $95 \%$ confidence intervals for ROC area were generated using a cluster bootstrapping technique (16). These confidence intervals were generated nonparametrically by randomly sampling from infants' predicted values and outcomes, with replacement, and recalculating the ROC area 500 times. The 2.5th and 97.5th percentile values of this sampling distribution were used as upper and lower bounds.

Assessment of Test Validity. We used a thresholdindependent approach to evaluating the models. For continuous measures such as a model output, a cutoff or threshold value must be chosen so that tests can be classified as positive or negative, and values of sensitivity, specificity, and predictive accuracies can be calculated. Although time-honored, this strategy has been severely questioned (17). It has the weakness of presuming that all physicians agree in the selection of threshold values. In fact, physicians might very well differ in their judgment on the basis of costs assigned to false-positive and false-negative results. Instead of imposing thresholds, we validated models by calculating the area under the ROC curve, which evaluates all possible threshold values. We evaluated models by calculating the fold increase in odds of an event as a variable is increased from the 25 th to 75 th percentile, and from the 10th to the 90th. For any patient at any point in time, we calculated the fold increase in odds of an event as exp(logit from dynamic model - logit from static model).

\section{RESULTS}

Study Population. Table 1 shows the demographic and heart rate characteristics of the 633 study infants in the derivation and validation groups. The higher median HRC index and lower SD and sample entropy at WFU suggested a higher risk for sepsis and sepsis-like illness at WFU, a referral-only center. The frequency of sepsis and sepsis-like illness was very similar at approximately $30 \%$. The most common blood culture result was no growth (123 of 155 at UVA and 91 of 118 at WFU). Coagulase-negative Staphylococcus was the organism most commonly isolated (16 at each center). Other pathogens isolated included Staphylococcus aureus, group B streptococcus, Escherichia coli, Enterococcus, Enterobacter, Klebsiella, Pseudomonas, and Candida. The most significant difference was in the mortality rate among infants with sepsis or sepsislike illness, which was nearly 3-fold higher at WFU.

The regression analyses were based on a derivation cohort at UVA, the training site, of 316 consecutive patients in whom there were 155 episodes of sepsis and sepsis-like illness in 110 patients. The validation cohort was 317 neonates in the WFU NICU, where there were 118 episodes of sepsis and sepsis-like illness in 93 infants.

Example of the HRC Analysis. Figure 1 shows representative 4096-beat time series of RR intervals from a single infant with the corresponding frequency histograms. Figure $1 A$ shows normal HRV, whereas Figure $1 B$ shows reduced variability with several transient, subclinical decelerations (i.e. the heart
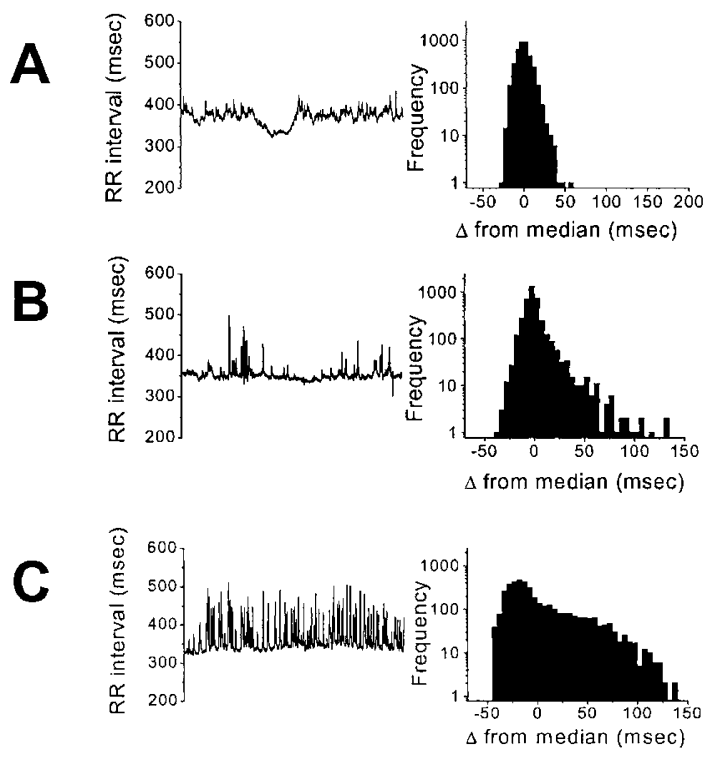

Figure 1. RR interval time series of length 4096 beats with normal $(A)$ and abnormal ( $B$ and $C$ ) HRC and the corresponding frequency histograms. Data in $B$ and $C$ were obtained 6 and $3 \mathrm{~h}$ before the clinical diagnosis of sepsis, respectively. Values of the mean RR interval $(\mathrm{ms})$ are $371(A), 353(B)$, and $363(C)$. The $\mathrm{SD}(\mathrm{ms})$ are $17.9(A), 15.0(B)$, and $34.6(C)$. The skewness values are $0.85(A), 1.49(B)$, and $1.11(C)$. The sample asymmetry values are $0.4(A)$, 9.6 $(B)$, and $12.2(C)$. The SampEn values are $0.90(A), 0.41(B)$, and $0.26(C)$. 
rate remained above 100 beats per minute, where RR intervals exceed $600 \mathrm{~ms}$ ), and Figure $1 C$ shows many decelerations. These are characteristic abnormalities of heart rate early in the course of sepsis (7) and present a challenge for classification because conventional measures of HRV such as the SD are similar in all three. As we reported previously, the skewness of the histograms are higher for the abnormal series in Figure 1, $B$ and $C$. In addition, they are further distinguished from normal by increased values of sample asymmetry and by decreased values of SampEn $(10,11)$.

Distributions of HRC. Figure 2 shows histograms of the HRC measures for the two sites. The smooth lines are Gaussian functions and support the hypothesis that the underlying distributions are normal or log-normal.

Changes in HRC with Gestational Age and Postmenstrual Age. Figure 3, $A-C$ shows regression-derived estimates of SD, SampEn, and sample asymmetry as a function of postmenstrual age. There were small but significant changes in each measure, with more abnormal values in the most premature infants. Analysis of interaction terms showed that postnatal changes were different in the most premature infants as well. As shown in Figure $3 D$, the HRC index is higher for premature infants at birth $(p<0.01)$. The interaction term was also significant, indicating that the shape of the curve varies across gestational age $(p<0.01)$. After approximately 250 postmenstrual days, there was no large difference.

Predictive Models for Neonatal Sepsis and Sepsis-Like Illness Using Clinical Information. The highest risk for neonatal sepsis and sepsis-like illness occurs in infants of lowest BW and GA, and the risk varies with time in the NICU. To guard against the possibility that our HRC measures were merely surrogates for these other, more conventional risk factors, we derived estimates of sepsis and sepsis-like illness in the next $24 \mathrm{~h}$ using BW, GA, and days of postnatal age as the predictor variables. As expected, these variables were associated with risk of sepsis and sepsis-like illness (Table 2). For example, a model trained and tested at UVA had ROC area 0.65 (model 1), and the same model tested at WFU had ROC area 0.67 (model 2). Because the demographic features of the
UVA and WFU NICUs are not the same, we derived a predictive model optimized for the WFU NICU population (model 3, ROC area 0.72).

Predictive Models for Neonatal Sepsis and Sepsis-Like Illness Using HRC. A regression model using only HRC parameters as predictor variables showed highly significant association with impending sepsis and sepsis-like illness at the training site (model 4). This regression equation was used to calculate the HRC index in all of the following tests. Importantly, HRC parameters added significantly to the predictive information of $\mathrm{BW}, \mathrm{GA}$, and days of age at the training site (model 5).

Validation of the Models. We found that the HRC index that was developed at UVA showed highly significant association with impending sepsis and sepsis-like illness at WFU (model 6). Again, HRC added significantly to the predictive information of BW, GA, and days of age (model 7). We also used the regression model 3 that optimized the relationships of $\mathrm{BW}$, GA, and days of age to sepsis and sepsis-like illness in the WFU NICU population. There was no large difference between the ROC areas of the models using HRC with either model based on demographic features $(0.77$ versus 0.75$)$. Thus predictive models developed at UVA, the training site, showed highly significant association with neonatal sepsis and sepsislike illness at WFU, the test site.

Evaluation of the Models. We evaluated the potential utility of the models by calculating the increase in odds of sepsis associated with a change in the model output. Figure 4 shows the fold increase in odds of sepsis and sepsis-like illness for infants at the 25th and 75th and at 10th and 90th percentiles of the model values. Static models based on demographics were useful in identifying infants at high risk for sepsis. Clinically, these models would be useful in comparing risks among infants. The dynamic models were also useful and could be equally applied in monitoring changing risks in an individual infant as well as in comparisons among infants.

Time Course of HRC in Neonatal Sepsis and Sepsis-Like Illness. Figure 5 shows time series plots of HRC-derived estimates of risk in two infants who had episodes of sepsis and
A
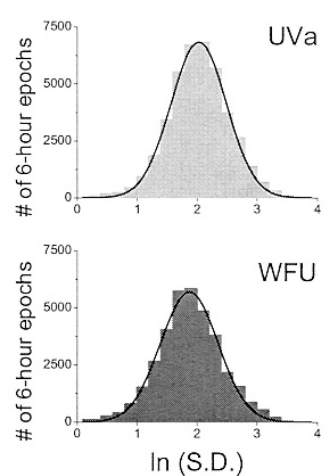

B
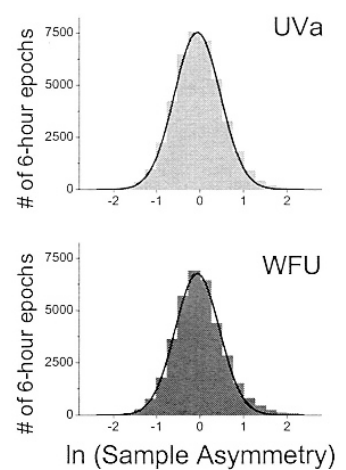

C
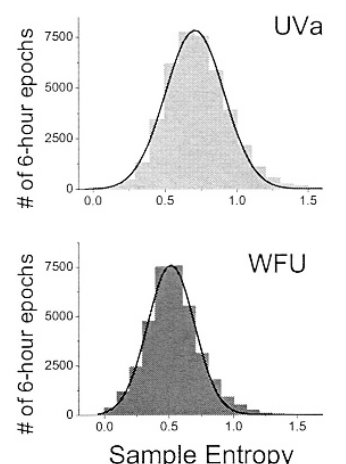

D
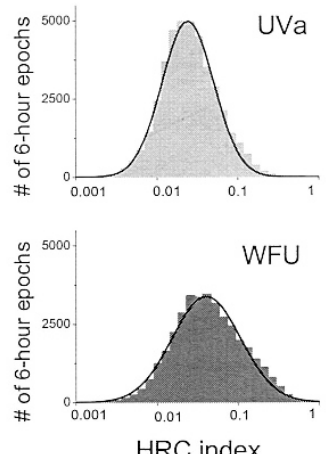

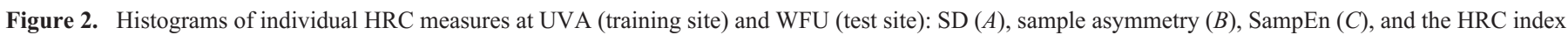

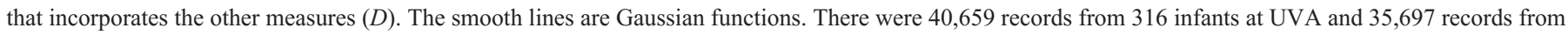
WFU. 

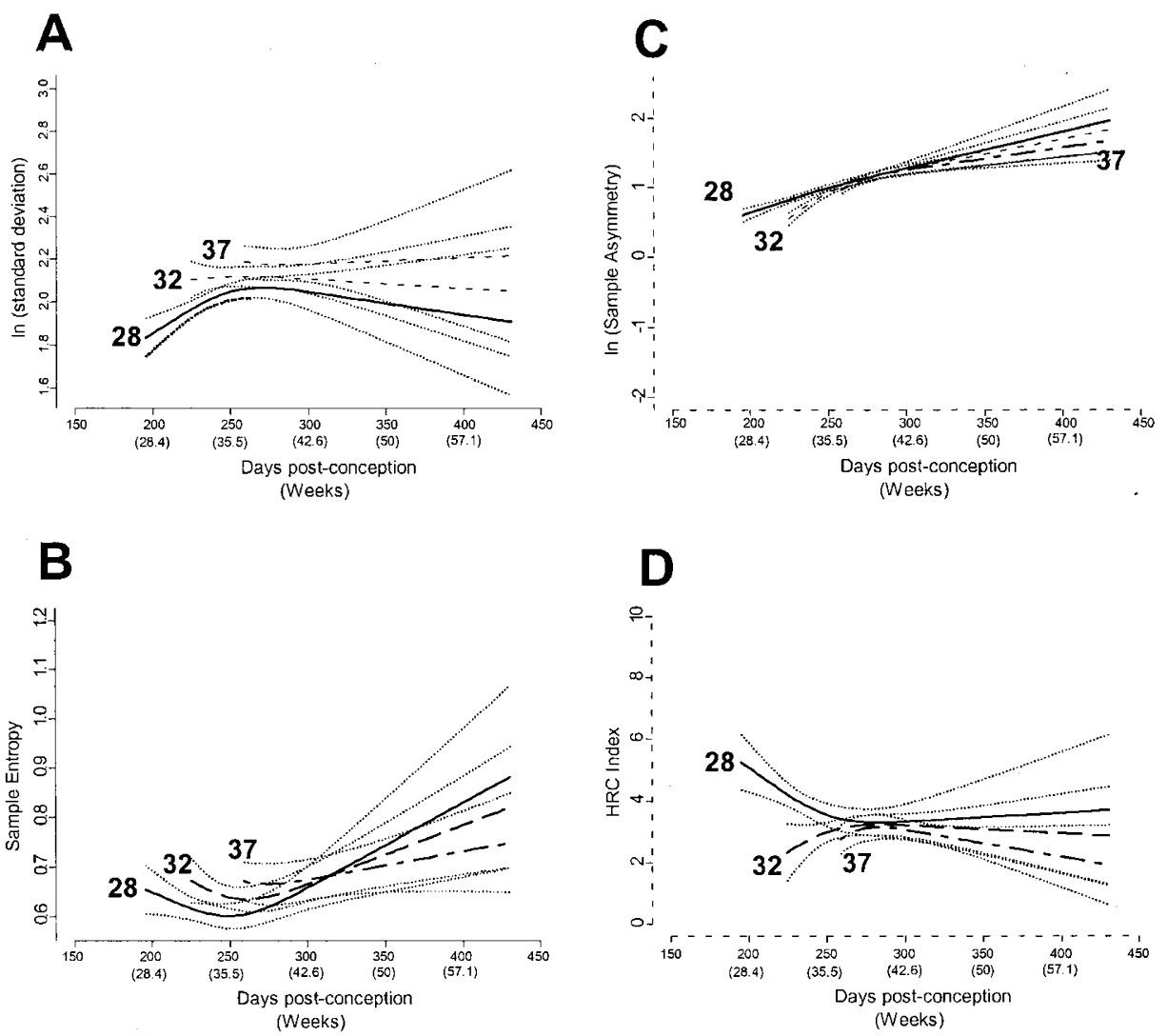

Figure 3. Changes in $\mathrm{HRC}$ as a function of postmenstrual age. The heavy lines represent data from the lowest quartile (GA 28 wk and less), middle half (median $32 \mathrm{wk}$ GA) and highest quartile (GA $37 \mathrm{wk}$ and greater). The dotted lines are $95 \%$ confidence intervals. HRV, as indexed by SD, was reduced in premature infants $(p<0.0001)$. The interaction term (GA * postmenstrual age) was also significant $(p=0.02)$, indicating a significant difference in the shape of the curve across GA. Sample asymmetry was significantly higher for premature infants $(p<0.001)$, and the difference in the shape of the curves was significant $(p<0.001)$. SampEn increased with increasing postmenstrual age after approximately $250 \mathrm{~d}(p=0.004)$. The rate of increase was greater among lower GA infants $(p=0.02$ for interaction term).

Table 2. Regression analysis of demographic features and HRC before neonatal sepsis and sepsis-like illness

\begin{tabular}{|c|c|c|c|c|c|c|c|c|c|c|}
\hline Model & Training site & $\begin{array}{l}\text { Test } \\
\text { site }\end{array}$ & Predictor & Predictor & Outcome & ROC area & $\chi^{2}$ & d.f. & $p 1$ & $p 2$ \\
\hline 1 & UVA & & demographics & & sepsis & 0.65 & 31 & 7 & $*$ & \\
\hline 2 & UVA & WFU & demographics & & sepsis & 0.67 & 20 & 1 & $*$ & \\
\hline 4 & UVA $\dagger$ & & & HRC & sepsis & 0.70 & 88 & 4 & $*$ & \\
\hline 5 & UVA & & demographics & $\mathrm{HRC}$ & sepsis & 0.72 & 99 & 11 & $*$ & * \\
\hline 6 & UVA & WFU & & HRC index & sepsis & 0.75 & 60 & 1 & * & \\
\hline
\end{tabular}

d.f., degrees of freedom in the regression model; demographics, GA, BW, and days of postnatal age; $p 1, p$ value for overall model; $p 2, p$ value for HRC or HRC index adding information to demographics.

WFU/UVA, models generated with demographic features from WFU and the single HRC index developed at UVA as predictor variables. Parameters of models without a test site are provided for purposes of describing the model and not for evaluation or validation.

$*<0.001$.

$\dagger$ The regression equation generated from this model yields the HRC index.

sepsis-like illness at the point labeled CRASH. The fold increase in odds of an event was calculated using the difference between the estimates of the static and dynamic models and represents the HRC-detected change in risk from that already expected to be present for an infant of given BW, GA, and days of postnatal age. When the value is near 1, the interpretation is that the HRC analysis detects no increased or decreased risk. In these two infants, one term (Fig. $5 A$ ) and the other premature (Fig. $5 B$ ), the HRC-derived estimate of risk rose intermittently over the $5 \mathrm{~d}$ before the event. Both infants recovered uneventfully with antibiotic therapy.

\section{DISCUSSION}

The main finding of this study is that regression models involving the HRC index, derived at the UVA NICU, were highly predictive of sepsis and sepsis-like illness at a NICU at a second site (WFU). At both sites, abnormal HRC of reduced 
A Static models

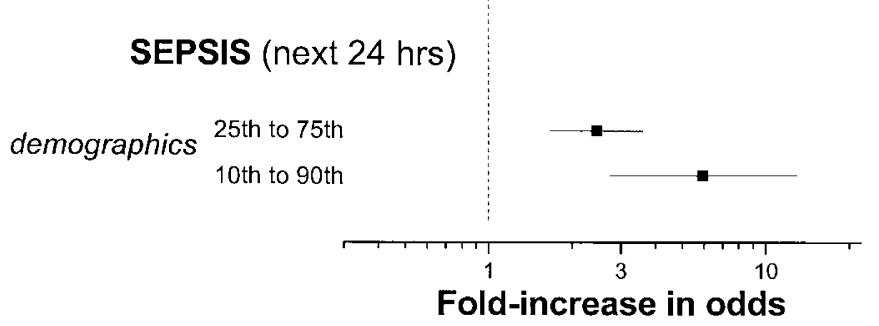

\section{Dynamic models}

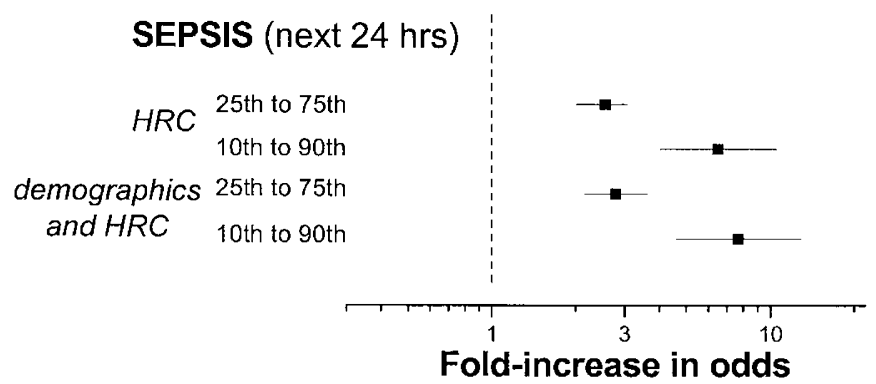

Figure 4. Fold increase in odds of neonatal sepsis and sepsis-like illness with changes in static and dynamic predictive models. Bars are 95\% confidence intervals.
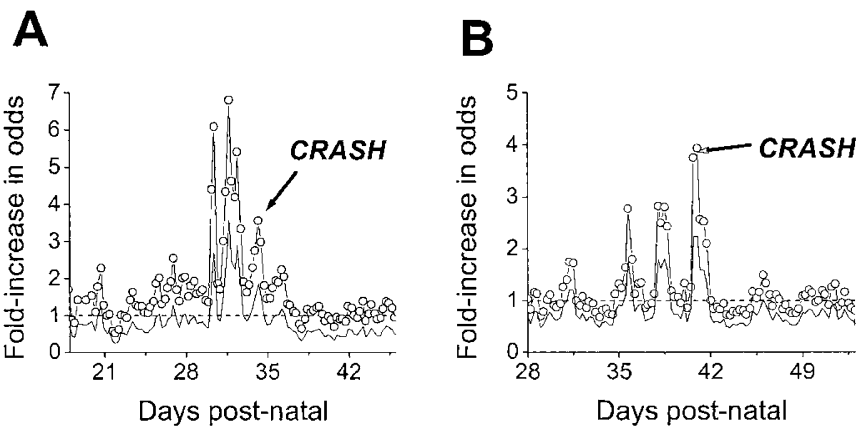

Figure 5. Fold increase in odds of neonatal sepsis and sepsis-like illness for two infants, each with an episode of sepsis at the point labeled CRASH (cultures, resuscitation, and antibiotics started here). The upper line is the fold increase in odds of event in the next $24 \mathrm{~h}$, and the lower line is the $95 \%$ lower confidence interval. $A$, The infant was term (GA $40 \mathrm{wk}$, BW $3000 \mathrm{~g}$ ), and a blood culture grew coagulase-negative staphylococci. The estimate of risk of an event was several-fold increased for up to $72 \mathrm{~h}$ before. $B$, The infant was premature (GA 26 wk, BW 660 g), a blood culture grew Staphylococcus aureus, and a urine culture grew E. coli.

variability and transient decelerations precede the clinical signs of sepsis and sepsis-like illness in newborn infants. We have previously developed novel measures of RR interval time series optimized to detect these abnormalities (7), which are observed before neonatal sepsis and sepsis-like illness as well as fetal distress. In this work, we used multivariable logistic regression to derive and to validate predictive models that used $\mathrm{HRC}$ and clinical demographic information to estimate the risk of upcoming illness and death.
We have evaluated the HRC index as a continuous measure of risk rather than as a test with a threshold. As described also by McIntire et al. (18), the risk of neonatal death was associated with decreasing birth weight, with no identifiable threshold. One way to present the information from the HRC index is as the fold change in risk of sepsis and sepsis-like illness over that expected from the known risk factors of BW, GA, and days of postnatal age. This kind of continuous measure may be readily interpretable by health care personnel and does not require a threshold for use in clinical care.

We note two major limitations of this study. First, the outcome of interest was driven by physician behavior. Although using the time of a blood culture for suspected sepsis has the advantage of unambiguity in time, it has the disadvantage that different physicians have different clinical judgment and behavior patterns, and thus the outcome measure has a degree of variability. Despite this arbitrary definition, however, the predictive models showed highly significant association of HRC changes with the physician's diagnosis of neonatal sepsis and sepsis-like illness. Second, we included episodes of illness associated with negative as well as positive blood cultures. We justify this approach on the basis of the similarity of HRC changes in infants with clinical deterioration regardless of culture result (7).

Infants at WFU, which admits only referral patients and has no labor and delivery facility, were at higher risk as judged from trends toward lower BW and higher mortality. These findings were recapitulated in the finding of more abnormal HRC measures at WFU, with reduced SD, decreased SampEn, and increased HRC index.

The mechanism of the HRC abnormality, which resembles that of fetal distress, is not known but might be traced to abnormalities of autonomic activity, circulating cytokines, or deprivation of cellular substrates. Distressed fetal sheep also have reduced variability and transient decelerations that can be elicited by hypoxia or acidosis and prevented by blockade of the parasympathetic nervous system with atropine (19). In sepsis, a possible mechanism is the presence of circulating cytokines, which interfere with signal transduction and are elevated before the clinical diagnosis of neonatal sepsis (20). The abnormal HRC of reduced variability and transient decelerations that we observe in newborn infants differs from that of critically ill children and adults with sepsis and systemic inflammatory response syndrome (SIRS) in which the only finding is reduced $\operatorname{HRV}(21,22)$. Current concepts of pathogenesis include uncoupling of autonomic and cardiovascular systems $(23,24)$, and we have suggested a cellular mechanism-dampened or interrupted intracellular signal transduction in sinus node cells (25). This mechanism might lead to both reduced variability and to transient decelerations. O'Rourke et al. $(26,27)$ showed that even mild substrate depletion in guinea pig heart cells leads to opening of ATPsensitive $\mathrm{K}$ channels and thus to membrane hyperpolarization and suppression of spontaneous depolarization. Episodes of substrate depletion in sino-atrial (SA) node pacemaker cells might be expected to lead to epochs of hyperpolarization and reduced firing rates. 
As shown in Figure 5, the HRC index-derived estimate of risk rose and fell intermittently over several days before the clinical diagnosis of sepsis. Although the pathophysiology is not known, such a pattern is consistent with the wellrecognized phenomenon of intermittent bacteremia (28-30).

Because HRC show small but significant changes postnatally, with findings consistent with higher risk in the most premature infants, the use of demographic features in the predictive models is justified. The increases in SD and sample asymmetry are consistent with increasing postnatal responsiveness of autonomic control of heart rate $(31,32)$. In particular, the increase in sample asymmetry reflects an increasing proportion of decelerations over accelerations, consistent with increasing vagal activity. Normally occurring episodes of neonatal decelerations (33) are distinguished from the abnormal episodes before neonatal sepsis in that the latter occur in the setting of reduced baseline variability.

Quantitative analysis of HRV has been studied extensively in adults with heart disease, and a consistent finding is that reduced variability is associated with poorer prognosis (34). Nonetheless, clinical use of HRV measures is not widespread, perhaps because adults with high-risk heart disease can already be identified using more conventional means, and no novel intervention can be readily suggested. Likewise, the finding of $\mathrm{T}$-wave alternans clearly identifies adults at high risk of sudden cardiac death $(35,36)$, but the therapy most likely to be effective, an implantable cardiac defibrillator, is expensive, invasive, and as yet untested for that indication.

Qualitative analysis of fetal heart rate characteristics, however, is very widely used clinically. Other than the reduction of neonatal seizures, there is no consistent demonstration of clinical utility (37), and the intervention involved, cesarean section, is expensive, invasive, and unproved to improve maternal or fetal outcome.

Where might quantitative analysis of neonatal HRC fit in? Such monitoring would be continuous, be noninvasive, and require no new contacts with the infant and is here shown to be associated with sepsis and sepsis-like illness, a major cause of morbidity and mortality. The intervention involved need only be timely assessment and closer observation by health care personnel, which is inexpensive. HRC monitoring may be an effective and continuous assessment strategy for the NICU with many high-risk infants.

Acknowledgments. We thank T. Smoot, N. Peters, K. Monahan, J. Richman, A. Dinn, W. King, S. Booth, and the physicians and nurses of the UVA and WFU NICUs for integral contributions to the data acquisition and analysis; and G. Beller, J. Kattwinkel, and B. Duling for support.

\section{REFERENCES}

1. Stoll BJ, Gordon T, Korones SB, Shankaran S, Tyson JE, Bauer CR, Fanaroff AA, Lemons JA, Donovan EF, Oh W, Stevenson DK, Ehrenkranz RA, Papile LA, Verte J, Wright LL 1996 Late-onset sepsis in very low birth weight neonates: a report from the National Institute of Child Health and Human Development Neonatal Research Network. J Pediatr 129:63-71

2. Escobar GJ 1999 The neonatal "sepsis work-up": personal reflections on the development of an evidence-based approach toward newborn infections in a managed care organization. Pediatrics 103:360-373
3. Gerdes JS, Polin RA 1987 Sepsis screen in neonates with evaluation of plasma fibronectin. Pediatr Infect Dis J 6:443-446

4. Philip AG, Hewitt JR 1980 Early diagnosis of neonatal sepsis. Pediatrics 65:1036-1041

5. Hammerschlag MR, Klein JO, Herschel M, Chen FC, Fermin R 1977 Patterns of use of antibiotics in two newborn nurseries. N Engl J Med 296:1268-1269

6. Garner JS, Jarvis WR, Emori TG, Horan TC, Hughes JM 1988 CDC definitions for nosocomial infections, 1988. Am J Infect Control 16:128-140

7. Griffin MP, Moorman JR 2001 Toward the early diagnosis of neonatal sepsis and sepsis-like illness using novel heart rate analysis. Pediatrics 107:97-104

8. Members of the ACCP/SCCM Consensus Conference Committee 1992 American College of Chest Physicians/Society of Critical Care Medicine Consensus Conference: Definitions for sepsis and organ failure and guidelines for the use of innovative therapies in sepsis. Crit Care Med 20:864-874

9. Bone RC, Grodzin CJ, Balk RA 1997 Sepsis: a new hypothesis for pathogenesis of the disease process. Chest 112:235-243

10. Richman JS, Moorman JR 2000 Physiological time series analysis using approximate entropy and sample entropy. Am J Physiol 278:H2039-H2049

11. Lake DE, Richman JS, Griffin MP, Moorman JR 2002 Sample entropy analysis of neonatal heart rate variability. Am J Physiol 283:R789-R797

12. Deleted in proof.

13. Kovatchev BP, Cox DJ, Gonder-Frederick LA, Young-Hyman D, Schlundt D, Clarke W 1998 Assessment of risk for severe hypoglycemia among adults with IDDM: validation of the low blood glucose index. Diabetes Care 21:1870-1875

14. Harrell FE, Pollock BG, Lee KL 1988 Regression models in clinical studies: determining relationships between predictors and response. J Natl Cancer Inst 80:1198-1202

15. Harrell FE, Margolis PA, Gove S, Mason KE, Mulholland EK, Lehmann D, Muhe L, Gatchalian S, Eichenwald HF 1998 Development of a clinical prediction model for an ordinal outcome: the World Health Organization Multicentre Study of Clinical Signs and Etiological Agents of Pneumonia, Sepsis and Meningitis in Young Infants. WHO/ARI Young Infant Multicentre Study Group. Stat Med 17:909-944

16. Feng Z, McLerran D, Grizzle J 1996 A comparison of statistical methods for clustered data analysis with Gaussian error. Stat Med 15:1793-1806

17. Guggenmoos-Holzmann I, van Houwelingen HC 2000 The (in)validity of sensitivity and specificity. Stat Med 19:1783-1792

18. McIntire DD, Bloom SL, Casey BM, Leveno KJ 1999 Birth weight in relation to morbidity and mortality among newborn infants. N Engl J Med 340:1234-1238

19. Yu ZY, Lumbers ER, Gibson KJ, Stevens AD 1998 Effects on hypoxaemia on foeta heart rate, variability and cardiac rhythm. Clin Exp Pharmacol Physiol 25:577-584

20. Kuster H, Weiss M, Willeitner AE, Detlefsen S, Jeremias I, Zbojan J, Geiger R, Lipowsky G, Simbruner G 1998 Interleukin-1 receptor antagonist and interleukin-6 for early diagnosis of neonatal sepsis 2 days before clinical manifestation. Lancet 352:1271-1277

21. Toweill D, Sonnenthal K, Kimberly B, Lai S, Goldstein B 2000 Linear and nonlinear analysis of hemodynamic signals during sepsis and septic shock. Crit Care Med 28:2051-2057

22. Korach M, Sharshar T, Jarrin I, Fouillot JP, Raphael JC, Gajdos P, Annane D 2001 Cardiac variability in critically ill adults: influence of sepsis. Crit Care Med 29:1380 1385

23. Ellenby MS, McNames J, Lai S, McDonald BA, Krieger D, Sclabassi RJ, Goldstein B 2001 Uncoupling and recoupling of autonomic regulation of the heart beat in pediatric septic shock. Shock 16:274-277

24. Godin PJ, Buchman TG 1996 Uncoupling of biological oscillators: a complementary hypothesis concerning the pathogenesis of multiple organ dysfunction syndrome. Crit Care Med 24:1107-1116

25. Nelson JC, Rizwan U, Griffin MP, Moorman JR 1998 Probing the order within neonatal heart rate variability. Pediatr Res 43:823-831

26. O'Rourke B, Ramza BM, Romashko DN, Marban E 1995 Metabolic oscillations in heart cells. Adv Exp Med Biol 382:165-174

27. O'Rourke B, Ramza BM, Marban E 1994 Oscillations of membrane current and excitability driven by metabolic oscillations in heart cells. Science 265:962-966

28. Zierdt CH 1983 Evidence for transient Staphylococcus epidermidis bacteremia in patients and healthy humans. J Clin Microbiol 17:628-630

29. Klein JO 2001 Bacterial sepsis and meningitis. In: Remington JS, Klein JO (eds) Infectious Diseases of the Fetus and Newborn Infant. WB Saunders, Philadelphia, pp 943-998

30. Squire E, Favara B, Todd J 1979 Diagnosis of neonatal bacterial infection: hematologic and pathologic findings in fatal and nonfatal cases. Pediatrics 64:60-64

31. Mazursky JE, Birkett CL, Bedell KA, Ben Haim SA, Segar JL 1998 Development of baroreflex influences on heart rate variability in preterm infants. Early Hum Dev 53.37-52

32. Sahni R, Schulze KF, Kashyap S, Ohira-Kist K, Fifer WP, Myers MM 2000 Maturational changes in heart rate and heart rate variability in low birth weight infants. Dev Psychobiol 37:73-81

33. Hodgman JE, Hoppenbrouwers T, Cabal LA 1993 Episodes of bradycardia during early infancy in the term-born and preterm infant. Am J Dis Child 147:960-964

34. Kleiger RE, Miller JP, Bigger Jr JT, Moss AJ 1987 Decreased heart rate variability and its association with increased mortality after acute myocardial infarction. Am J Cardiol 59:256-262

35. Gold MR, Bloomfield DM, Anderson KP, El Sherif NE, Wilber DJ, Groh WJ, Estes NA, Kaufman ES, Greenberg ML, Rosenbaum DS 2000 A comparison of T-wave alternans, signal averaged electrocardiography and programmed ventricular stimulation for arrhythmia risk stratification. J Am Coll Cardiol 36:2247-2253

36. Rosenbaum DS, Jackson LE, Smith JM, Garan H, Ruskin JN, Cohen RJ 1994 Electrical alternans and vulnerability to ventricular arrhythmias. N Engl J Med 330:235-241

37. Thacker SB, Stroup DF, Peterson HB 1995 Efficacy and safety of intrapartum electronic fetal monitoring: an update. Obstet Gynecol 86:613-620 